\title{
11 p11.12p12 duplication in a family with intellectual disability and craniofacial anomalies
}

\author{
Xuejiao Chen ${ }^{1 \dagger} \odot$, Huihui X ${ }^{1 \dagger}{ }^{\dagger}$, Weiwu Shi ${ }^{1 \dagger}$, Feng Wang ${ }^{2}$, Fenfen $X u^{3}$, Yang Zhang ${ }^{1}$, Jun Gan ${ }^{1}$, Xiong Tian ${ }^{4}$, \\ Baojun Chen ${ }^{5}$ and Meizhen Dai ${ }^{*}$
}

\begin{abstract}
Background: Potocki-Shaffer syndrome (PSS) is a rare contiguous gene deletion syndrome marked by haploinsufficiency of genes in chromosomal region 11p11.2p12. Approximately 50 cases of PSS have been reported; however, a syndrome with a PSS-like clinical phenotype caused by 11p11.12p12 duplication has not yet been reported.
\end{abstract}

Methods: 11p11.12p12 duplication syndrome was identified and evaluated using a multidisciplinary protocol. Diagnostic studies included intelligence testing, thorough physical examination, electroencephalography (EEG), magnetic resonance imaging (MRI) of the brain, ultrasonography, biochemical tests and karyotype analysis. Next-generation sequencing analysis clarified the location of the chromosomal variations, which was confirmed by chromosome microarray analysis (CMA). Whole-exome sequencing (WES) was performed to exclude single nucleotide variations (SNVs). A wider literature search was performed to evaluate the correlation between the genes contained in the chromosomal region and clinical phenotypes.

Results: The proband was a 36-year-old mother with intellectual disability (ID) and craniofacial anomalies (CFA). She and her older son, who had a similar clinical phenotype, both carried the same 11p11.12p12 duplication with a copy number increase of approximately $10.5 \mathrm{Mb}$ (chr11:40231033_50762504, GRCh37/hg19) in chromosome bands $11 \mathrm{p} 11.12 \mathrm{p} 12$. In addition, she gave birth to a child with a normal phenotype who did not carry the 11p11.12p12 duplication. By literature research and DECIPHER, we identified some shared and some distinct features between this duplication syndrome and PSS. One or more of ALX4, SLC35C1, PHF21A and MAPK8IP1 may be responsible for 11 p11.12p12 duplication syndrome.

Conclusions: We present the first report of $11 \mathrm{p} 11.12 \mathrm{p} 12$ duplication syndrome. It is an interesting case worth reporting. The identification of clinical phenotypes will facilitate genetic counselling. A molecular cytogenetic approach was helpful in identifying the genetic aetiology of the patients and potential candidate genes with triplosensitive effects involved in 11 p11.12p12 duplication.

Keywords: 11p11.12p12 duplication, Intellectual disability, Chromosome 11, Molecular cytogenetics, Genetic counselling

*Correspondence: daimz@enzemed.com

${ }^{\dagger}$ Xuejiao Chen, Huihui Xu and Weiwu Shi contributed equally to this work.

${ }^{1}$ Medical Research Center, Taizhou Hospital of Zhejiang Province

Affiliated to Wenzhou Medical University, Linhai, Zhejiang, China

Full list of author information is available at the end of the article

\section{Background}

With the increasing clinical application of molecular cytogenetics, such as next-generation sequencing analysis and chromosome microarray analysis (CMA), increasing numbers of chromosomal deletions/microdeletions 
and duplications/microduplications have been detected and identified, affording new development opportunities for genetics and new challenges for clinical genetic counselling, especially prenatal genetic counselling. PotockiShaffer syndrome (PSS) is caused by a rare contiguous gene deletion in chromosomal region 11p11.2p12 (chr11: 43421550_48821552, GRCh37/hg19). Our patient's clinical phenotype is also similar but not entirely identical to that of PSS; however, a syndrome caused by 11p11.12p12 duplication has not yet been reported. Here, we report a de novo $11 \mathrm{p} 11.12 \mathrm{p} 12$ duplication in a small family presenting with intellectual disability (ID) and craniofacial anomalies (CFA). The duplication encompasses approximately $10 \mathrm{Mb}$ (chr11: 40231033_50762504, GRCh37/ hg19), which cannot be distinguished by G-banding 400band resolution in karyotype analysis; therefore, it may also be called a microduplication.

The most common phenotype caused by chromosomal abnormalities is ID, which affects approximately $1 \%$ of the population [1]. There are approximately 1900 high-/moderate-confidence ID-causing genes that are annotated in the Genomics England ID panel and DDG2P (https:// www.ebi.ac.uk/gene2phenotype). The gene-disease identities of 484 genes (including many non-ID genes) have been curated by ClinGen (https://www.clinicalgenome. org/) [2]. The organ specificity of four genes involved in the 11p11.12p12 chromosomal region, namely, SLC35C1, PEX16, PHF21A, and RAPSN, is considered in DDG2P to be related to brain/cognition, and the patients' phenotypes suggest that one or more genes that likely contribute to the clinical phenotype in this syndrome are located in the duplicated region.

\section{Methods}

\section{Karyotype analysis}

Karyotype analysis was performed in 6 main members of the family to identify the genetic aetiology. Chromosomes were obtained from cultured peripheral lymphocytes. The conventional technique of $\mathrm{G}$ banding analysis was used. Twenty-five metaphases of the family members were analysed at 550 chromosome band resolution. The International System for Human Cytogenomic Nomenclature (ISCN, 2016) served as the reference for describing the chromosomes.

\section{Next-generation sequencing analysis}

To clarify the location of the chromosomal variations and exclude normal chromosomal variations, 11 members of the family underwent next-generation sequencing analysis, which was also performed in 7270 pregnant women to detect chromosomal microdeletions and microduplications for prenatal diagnosis. DNA was extracted from patient whole blood or amniotic fluid using a MagPure
Universal DNA LQ Kit. A DNA library was obtained after the DNA was fragmented, end-repaired, linkerligated and PCR-amplified. Then, the library DNA was sequenced using a BioelectronSeq 4000 instrument. The kits used in this study included the Ion Plus Fragment Library Kit, the Ion PI Hi-Q OT2 200 Templating Kit, the Ion PI Hi-Q 200 Sequence Kit and the Ion PI Chip V3. All reagents were provided by Dongguan BoaoMuhua Gene Technology Co, Ltd, China.

\section{Chromosome microarray analysis}

CMA was used to confirm the location of the chromosomal variations in the family and detect chromosomal microdeletions and microduplications in 2906 pregnant women for prenatal diagnosis. Human genomic DNA was extracted from whole blood or amniotic fluid using a QIAamp DNA Mini Kit. The following standard experimental procedures were performed: digestion, ligation, polymerase chain reaction (PCR), PCR purification, fragmentation, labelling, hybridization, washing, staining, and scanning. The instrument was an Affymetrix GeneChip System 3000D x v.2 chip system. The CytoScan $750 \mathrm{~K}$ Array includes 200,000 genotype-able SNPs and 550,000 non-polymorphic probes. All genotyping and copy number analysis were performed with Chromosome Analysis Suite (ChAS) Software version 3.0. All data were required to pass quality control (QC) metrics, including the median of the absolute values of all pairwise differences $\leq 0.25$, SNPQC $\geq 15$, and a waviness standard deviation $\leq 0.12$.

\section{Whole-exome sequencing (high-throughput testing)}

Whole-exome sequencing (WES) was performed to exclude single nucleotide variations (SNVs). Genomic DNA was extracted using a QIAamp DNA Extraction Kit. The extracted DNA was fragmented with DNase and purified by magnetic beads, followed by PCR amplification and ligation of the adaptor sequence, which was twice captured and purified by a TruSight One Sequencing Panel (Illumina Inc, USA) and then amplified by PCR. The final library obtained after purification was sequenced in the exon regions of 4811 clinically relevant genes in a MiSeq sequencer (Illumina Inc, USA). The TruSight One Sequencing Panel is based on the Human Gene Mutation Database (HGMD Professional), the Online Mendelian Inheritance in Man (OMIM), the GeneTests website (www.genetests.org), and others from Illumina. Information about the commercial kits that ultimately incorporate genes relevant to diabetes-related gene sequencing is provided by the manufacturer (http:// www.illumina.com/products/trusight-one-sequencingpanel.ilmn). 
All the data were compared to the reference sequence (UCSC hg19) using the Burrows-Wheeler Aligner (BWA; http://bio-bwa.sourceforge.net/) algorithm. In the screening process, the clinical data were combined with bioinformatics software (PolyPhen2, LRT, Mutation Taster, etc.) to predict the results, function, variation and genetic model of each gene; these results were analysed to identify suspicious candidate mutations, which were verified by Sanger sequencing. PCR primers were designed for the sites of suspected candidate mutations. The corresponding sites of the parents' genomes were detected.

\section{Results}

\section{Clinical features}

The proband (II4, Fig. 1a) was a 31-year-old pregnant woman. She was $154 \mathrm{~cm}$ in height (-1.22 SDs), shorter than the mean height $(160 \mathrm{~cm})$ of the women in the family by $6 \mathrm{~cm}$. She showed mild ID, occasional dizziness, resting tremor, and CFA (Fig. 1d). Her Wechsler Adult Intelligence Scale (WAIS) test score was $I Q=56$. She could take care of herself but could not work normally while chatting with others. Her language expression exhibited some difficulties; for example, she could not accurately read the number 888 . She also had difficulty adding numbers from 1 to 10 .

We performed a karyotype analysis (G-banding-550 bands), and the result was $46, \mathrm{XX}, \operatorname{dup}(11)(\mathrm{p} 11.12 \mathrm{p} 12)$ (Fig. 1b). In order to further clarify the location of the chromosome 11 duplications, CMA and nextgeneration sequencing analysis was performed. The results of sequencing analysis revealed a copy number increase of approximately $10.53 \mathrm{Mb}$ in chromosome bands 11p11.12-p12 ranging from nucleotides 40231033_50762504 (Fig. 2a), a copy number loss of approximately $292.18 \mathrm{~kb}$ in chromosome bands $2 \mathrm{q} 11.2$, and a copy number increase of approximately $104.94 \mathrm{~kb}$ in chromosome bands 16p11.2 (Table 1). Microduplication of $16 \mathrm{p} 11.2$ was detected in 1 of 10,176 foetuses, with a normal phenotype based on follow-up, whereas the $11 \mathrm{p} 11.12 \mathrm{p} 12$ duplication and $2 \mathrm{q} 11.2$ microdeletion were not found among these 10,176 foetuses (Table 1). In addition, microdeletion of $2 \mathrm{q} 11.2$ and microduplication of $16 \mathrm{p} 11.2$ involve no pathogenic genes, with full coverage of polymorphisms in the Database of Genetic Variants (DGV). The 11p11.12p12 duplication was confirmed by a third experiment involving CMA (Fig. 2b). We also collected peripheral blood from the patients' parents and performed karyotype analysis to determine the source of

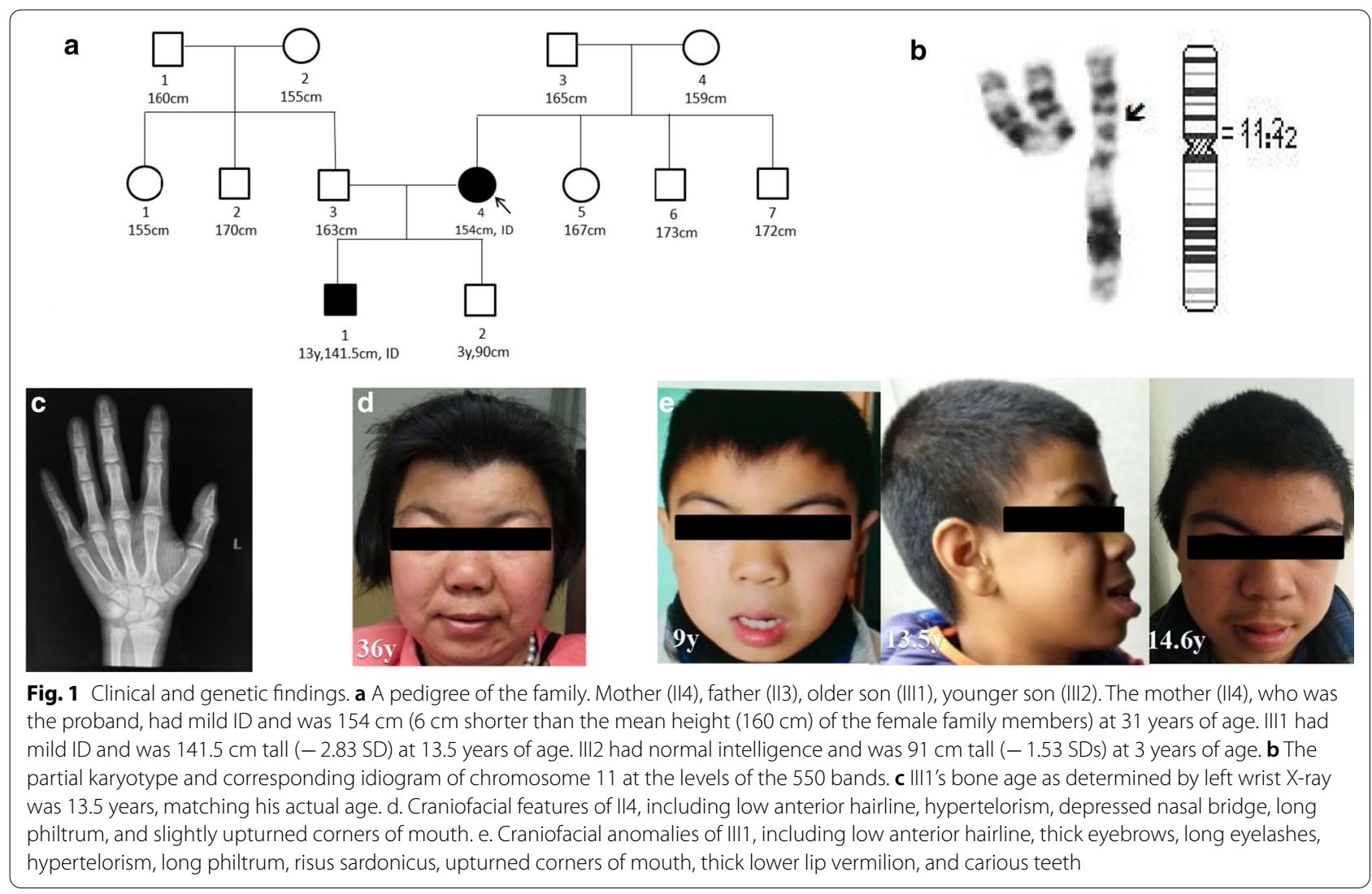




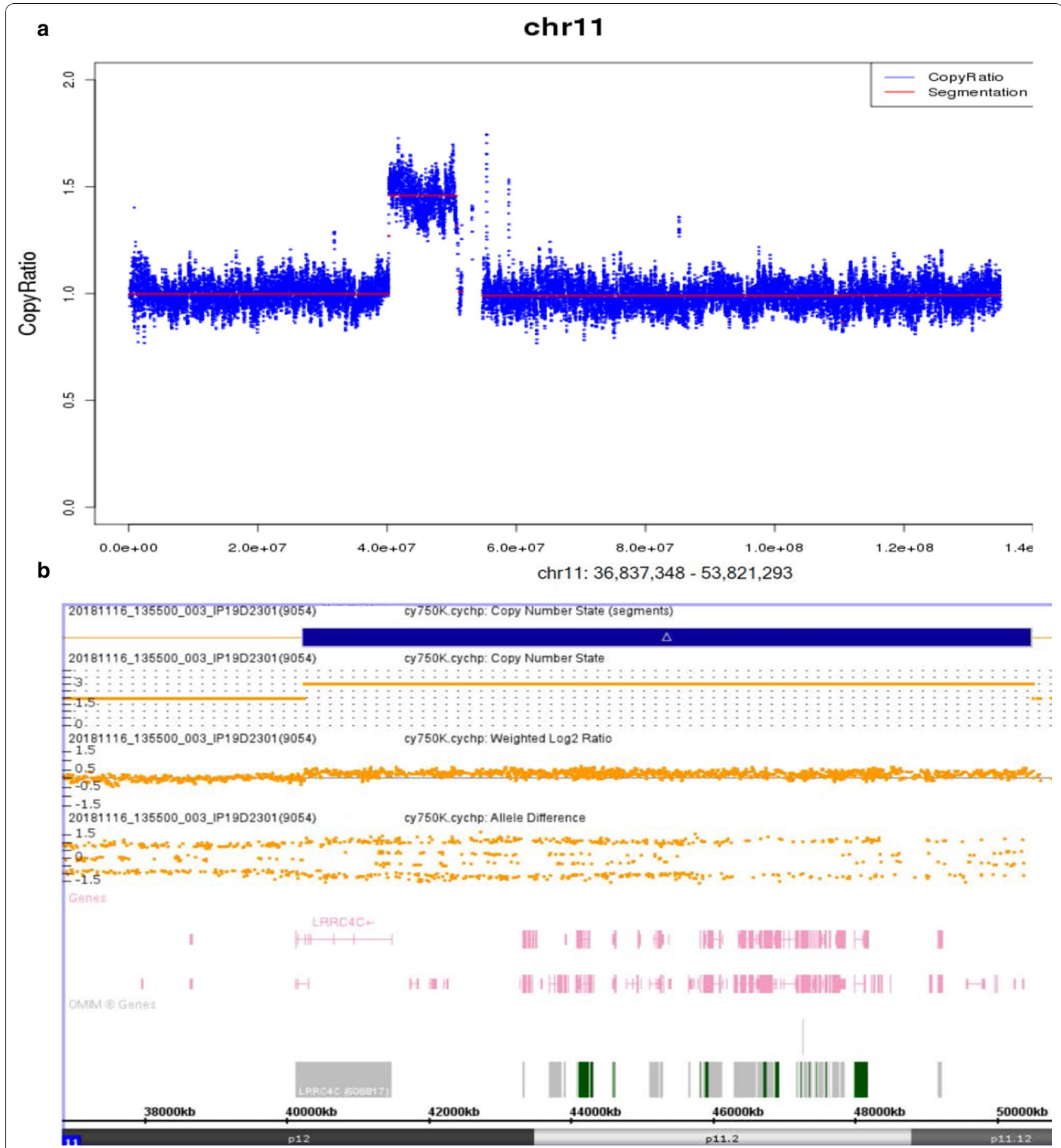

Fig. 2 Molecular details. a A next-generation sequencing profile of the 10.53-Mb interstitial duplication at 11p11.12p12 in II4. b CMA profile of the 10.26-Mb duplication at 11p11.12p12 in II4, the same as in III1

the 11p11.12p12 duplication. The chromosome karyotypes of the parents were normal (Table 1).

At 36 years, the proband's physical examination in the neurology department showed resting tremor in both hands, which was obvious in the right hand. She had experienced confusion twice in 15 years. The electroencephalogram (EEG) results revealed moderate abnormalities, a small number of spikes as low as 110 microvolts and sharp slow waves were scattered, with the temporal region as the focus. Therefore, she was suspected to have 


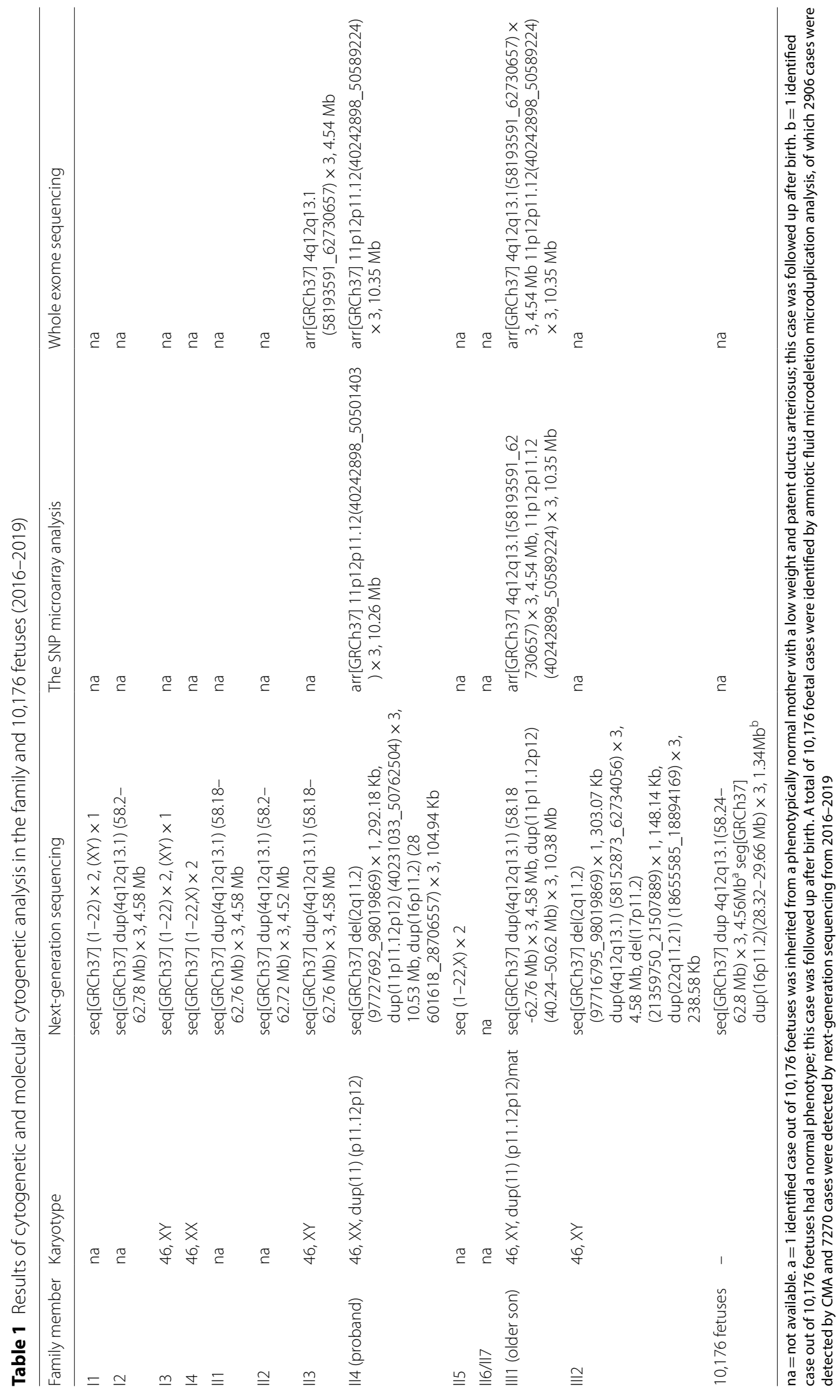


seizures. Chest computed tomography (CT) displayed two cumulative changes in the lungs. Magnetic resonance imaging (MRI) of the brain showed abnormal signals of the centrum semiovale on both sides and a diffuse corona radiata, which suggested the possibility of demyelinating lesions. The analyses of lymphocyte subsets and immunoglobulin levels were normal.

III1 was the older son of the proband (Fig. 1e). His clinical phenotype included mild ID, suspected seizures, short stature, and CFA. He walked at 15 months. His first words were at 2 years. At 9 years, his neuropsychiatric evaluation revealed mild cognitive delay (IQ of 67 using the WISC-IV), speech delay and learning difficulties. His computational ability was poor; he could calculate one plus one but could not correctly calculate two plus two. He could write only approximately 100 simple words and could put fewer than three words together. In terms of self-care ability, he could neither correctly wear complicated clothes nor distinguish the left from the right shoe. Routine blood tests showed that red blood cell counts and haemoglobin content were slightly lower than normal. The results of trace element analysis and biochemical tests were normal. At 10 years, he could correctly distinguish the left from the right shoe but could still not distinguish between his left and right hands.

At the age of 13.5 years, he was $141.5 \mathrm{~cm}(-2.83 \mathrm{SDs})$. His bone age was also 13.5 years according to left wrist $\mathrm{X}$-ray (Fig. 1c). His testicular volume was approximately $12 \sim 15 \mathrm{ml}$ (normal). His pubic hair was in stage PH1 (normal), and his growth hormone value was also normal at $14.1 \mathrm{ng} / \mathrm{ml}$ (reference value $<5.0 \mathrm{ng} / \mathrm{ml}$ ). These data suggest that his developmental indicators were typical for his age of 13.5 years, but his height is significantly lower than the height of $13.5 \mathrm{y}$. Though his computing power and language skills had improved, he could still not speak in sentences of more than 5 words, and he could still not distinguish his left and right hands. He could correctly add 2 plus 2 or 4 plus 4 by memorization but still could not correctly add 1 plus 3 .

At 14.6 years, physical examination in the neurology department revealed appendicular hypotonia and abnormal gait. Approximately a year ago, he had fallen asleep and could not be awakened for an hour. He was given an EEG examination, which displayed mild to moderate abnormalities, and small numbers of spiking waves as low as 90 microvolts were seen in the left posterior temporal and occipital regions. Thus, he was suspected seizures. Medical examinations, including echocardiographic examination, renal and urinary tract ultrasound, lymphocyte subset and immunoglobulin level analysis, chest CT, and MRI of the brain, were normal.

The karyotype analysis result of III1 was 46, XY, dup (11)(p11.12p12) mat. Molecular cytogenetic analysis of III1 revealed two duplications with a copy number increase in chromosome bands 11p11.12p12 (10.38 Mb) inherited from his mother and a copy number increase in chromosome bands 4q12q13.1 (4.58 Mb) inherited from his father. Moreover, 4q12q13.1 microduplication was found in one of 10,176 foetuses, with a low weight and patent ductus arteriosus; based on follow-up, the microduplication was inherited from a normal-phenotype mother. It was also found in 5 normal-phenotype members in the family (Table 1). To exclude abnormalities in other genes outside the 11p11.12p12 interval causing the phenotype of this family, we performed WES in II3, II4, and III1. No other gene anomalies were found (Table 1).

III2 was the younger son of the proband. During the second trimester, amniotic fluid karyotype analysis and next-generation sequencing analysis were performed. The results showed two microduplications and two microdeletions (Table 1). Del (2q11.2) and dup (4q12q13.1) were inherited from his mother and father, respectively. Del (17p11.2) and dup (22q11.21) were de novo copy number variations (CNVs), which were not found among 10,176 foetuses yet. Not all of the CNVs involving pathogenic genes were found in OMIM but were fully covered by GDV. No cases with phenotypes in the same interval of del (17p11.2) and dup (22q11.21) were found in the Database of Chromosomal Imbalance and Phenotype in Humans Using Ensembl Resources (DECIPHER). His karyotype was also normal. The results indicated that the foetus had no pathogenic chromosomal abnormalities, and the pregnancy continued to deliver. He began to learn to walk and vaguely said the word "dad" at 1 year old. He exhibited no significant difference compared with normal children in infancy and childhood. His height was $91 \mathrm{~cm}(-1.53 \mathrm{SDs})$, and the difference between the younger son and the mean height of the family was -0.24 SDs. No significant differences in chromosome karyotype, developmental delay or ID were observed for the other members of the family.

\section{Discussion}

With the exception of the 11p11.12p12 duplication, none of the microdeletions or microduplications in the pedigree contain the pathogenic genes included in OMIM and are fully covered by DGV. These microdeletions and microduplications were also observed in members of the family or other foetuses with a normal phenotype, although the 4q12q13.1 microduplication segments were longer than $4 \mathrm{Mb}$. A total of 11p11.12p12 segments containing 100 genes were detected only in the patient and her older son with the clinical phenotype. These findings suggest that the clinical phenotype of this family is caused by duplication of the chromosome 11 region. The 5-year follow-up data after the birth of the child also showed 
that the 11p11.12p12 duplication was the cause of the disease in this family, and our accurate genetic analysis of prenatal diagnosis provided the correct genetic counselling for the child.

Potocki-Shaffer syndrome (OMIM 601224) is a rare contiguous gene deletion syndrome due to haploinsufficiency of genes in chromosomal region 11p11.2p12 (chr11:43421550_48821552, GRCh37/ hg19), similar to the segments duplicated in our patient (chr11:40231033_50762504Mb, GRCh37/hg19). Although the duplication interval $(10 \mathrm{Mb})$ of our case was significantly larger than the PSS interval (5.4 Mb), there was no difference in the phenotypic genes they contained. PSS patients are very rare, with no more than 50 cases described in the literature. To determine the correlation between the genes contained in this chromosomal region and clinical phenotypes, we searched the clinical phenotypes of all PSS patients in an attempt to identify common characteristics. This syndrome is characterized by CFA, developmental delay, multiple exostoses, biparietal foramina, and ID [3-17]. PSS occasionally manifests as epilepsy, hypotonia and central nervous system anomalies [5-7, 9, 11-13, 17]. Craniofacial abnormalities include brachycephaly [8-10, 17], microcephaly $[10-12,17]$, bilateral parietal foramina $[5,6,12,15,17]$, broad forehead $[8,10]$, high forehead $[5,7,9,13]$, laterally sparse eyebrows $[7,12,15,17]$, upslanting/downslanting palpebral fissures $[5,7,10]$, bilateral epicanthal folds $[6,8$, $10,13,14,17]$, left ptosis [12, 14], esotropia [5, 6], hypertelorism [10, 13, 16], hypotelorism [10], mid-facial hypoplasia $[11,15]$, narrowed nasal bridge $[10,14,15,17]$, depressed nasal bridge $[9,10]$, broad nasal bridge $[8,16]$, short philtrum $[6,7,14,17]$, thin lips $[7,8,10,13]$, downturned mouth angle [5-7], dysplastic low-set ears [5, 10, $13]$, and mild micrognathia $[6,8,10]$. Most of the overlapping phenotypes appear in more than 3 studies and are most likely caused by $11 \mathrm{p} 11.2 \mathrm{p} 12$ deletions. We also searched cases of chromosomal duplications or microduplications in the same chromosomal region, which were found only in the DECIPHER (Table 2). Most of the characteristics of our patient are consistent with those described in the DECIPHER database (Fig. 3). The most common clinical features of chromosome duplications in the $11 \mathrm{p} 11.12 \mathrm{p} 12$ region are hypertelorism, ID, and thick eyebrows, short stature, followed by long eyelashes, EEG abnormalities, speech and expressive language delay, and cognitive impairment. The minimal clinical feature was ID, and the chromosome position was concentrated between 45427775 and 46949520 (GRCh38), a region that contains the SLC35C1, MAPK8IP1, PEX16, CREB3L1, $Z N F 408, F 2$, and $L R P 4$ genes. The critical region involved in PSS was also 11p11.2, spanning $2.1 \mathrm{Mb}$ between D11S1393 to D11S1385/D11S1319 (44.6-46.7 Mb), and the gene related to ID may be located between 45.6 and $46.7 \mathrm{Mb}$ [17]. Overall, there are common and distinct features between 11p11.2p12 deletion and duplication. The common clinical features include ID, hypertelorism, Micrognathia, hypotonia, Global developmental delay, and seizures. The distinct clinical features include long philtrum, low anterior hairline, thick eyebrows, upturned corners of mouth, and thick lower-lip vermilion (Table 2). The similarities and differences suggest that gene-dosage effects are not all linearly superimposed, especially in the development of the nervous system. The normal range of gene-dosage effects seems to be very narrow, similar to the equivalent zone of antigen-antibody reaction, and more or less doses will not be acceptable.

We know that $11 \mathrm{p} 11.12 \mathrm{p} 12$ encompasses approximately 18 morbid genes, including EXT2 (OMIM*608210), ALX4 (OMIM*605420), CD82 (OMIM*600623), SLC35C1 (OMIM*605881), MAPK8IP1 (OMIM*604641), MYBPC3 (OMIM*600958), PHF21A (OMIM*608325), LRP4 (OMIM*604270), CREB3L1 (OMIM*616215), ZNF408 (OMIM*616454), F2 (OMIM*176930), DDB2 (OMIM*600811), ACP2 (OMIM*171650), SLC39A13 (OMIM*608735), RAPSN (OMIM*601592), PEX16 (OMIM*603360), NDUFS3 (OMIM*256000), and PTPRJ (OMIM*600925). Which genes within the interval contribute to $11 \mathrm{p} 11.12 \mathrm{p} 12$ duplication syndrome and even PSS remains to be determined. EXT2 is an important pathogenic gene in PSS because its mutation mainly causes multiple exostoses. ALX4 is expressed in various organs and plays an essential role in the development of the skull, limbs, and skin [18]. ALX4 mutations result in functional haploinsufficiency, such as the development of frontonasal dysplasia 2, impaired interfollicular epidermal differentiation and perturbed regular hair follicle differentiation $[19,20]$. This gene may be responsible for some of our patient's craniofacial abnormalities. The SLC35C1 gene encodes a GDP-fucose transmembrane transporter (FucT1) located in the Golgi apparatus [21]. It is abundantly expressed in the brain, gastrointestinal tract, female tissues, male tissues, kidney and urinary bladder, bone marrow, and lymphoid tissues. Furthermore, RNA tissue-specific expression is enhanced in the liver in HPA (https://www.proteinatlas.org). SLC35C1 is a negative regulator of Wnt signalling in colon cancer. In fact, overexpressing SLC35C1 inhibits the canonical Wnt pathway [22]. SLC35C1 heterozygous mutations may cause partial in vivo defects in fucosylation [23]. The PHF21A gene encodes BHC80, a component of the BRAF35/histone deacetylase complex (BHC), which mediates repression of neuron-specific genes through the cis-regulatory element known as repressor element-1 (RE1) or neural restrictive silencer (NRS). Based on RT-PCR, the highest level of tissue-specific expression 


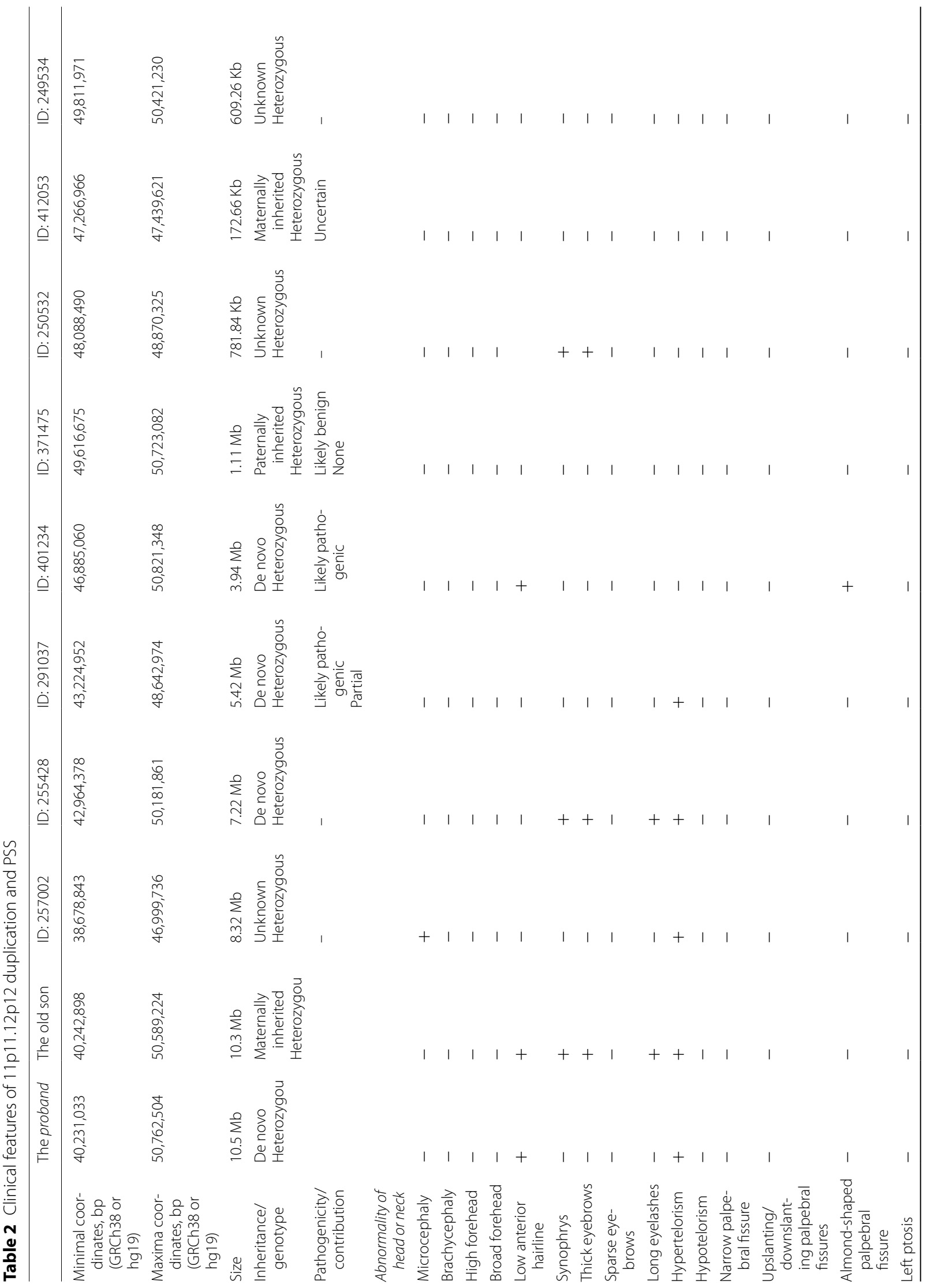




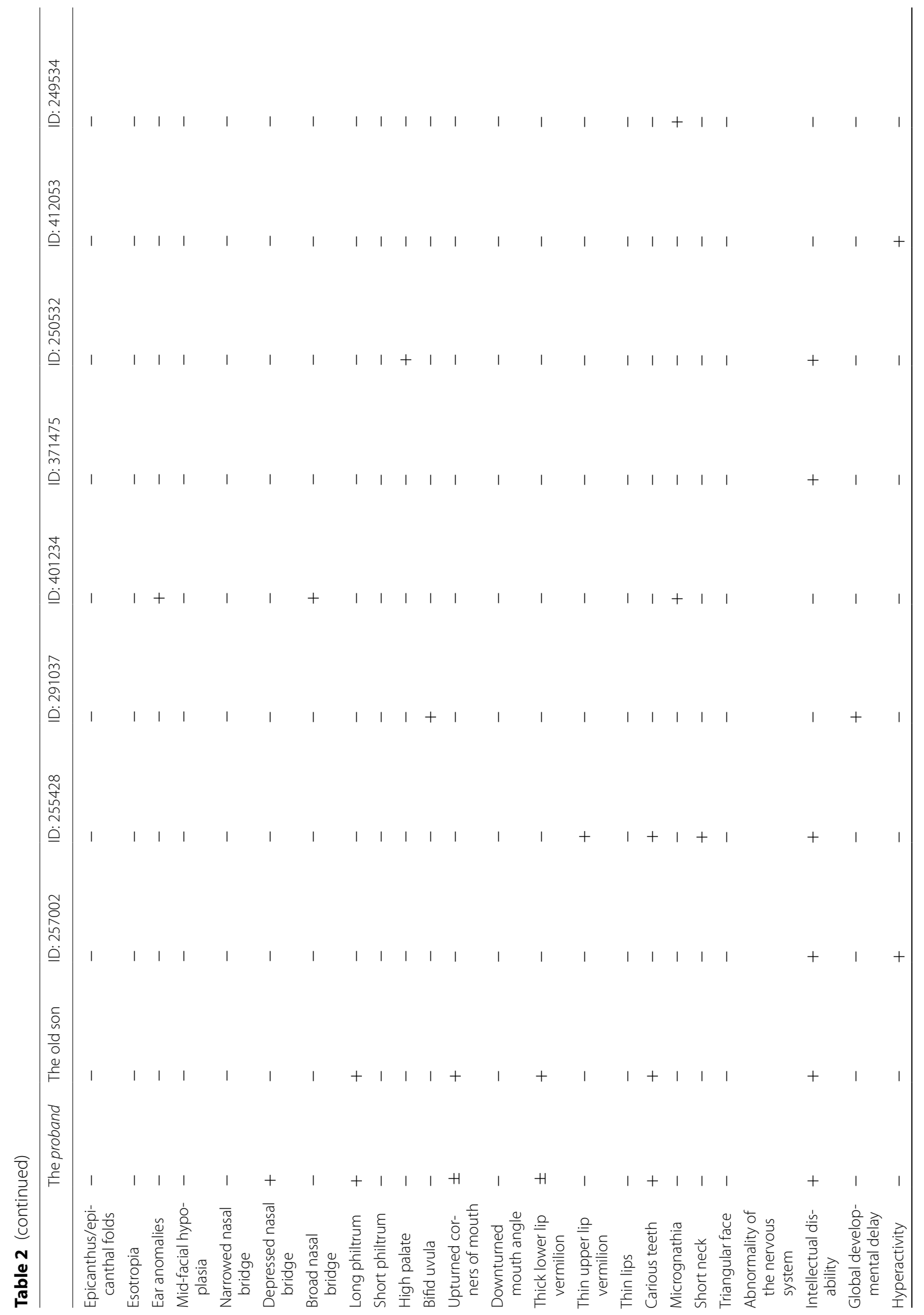




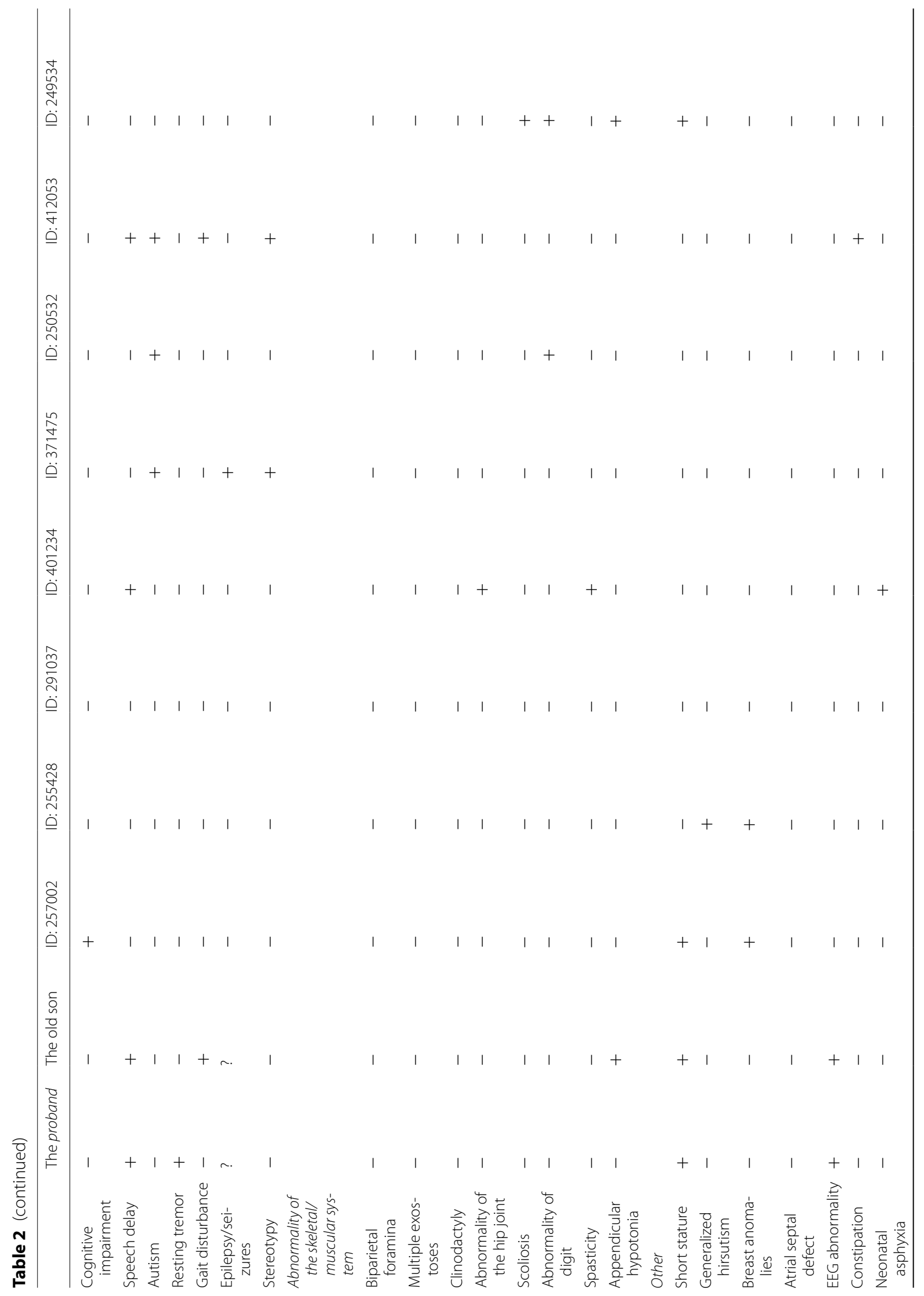




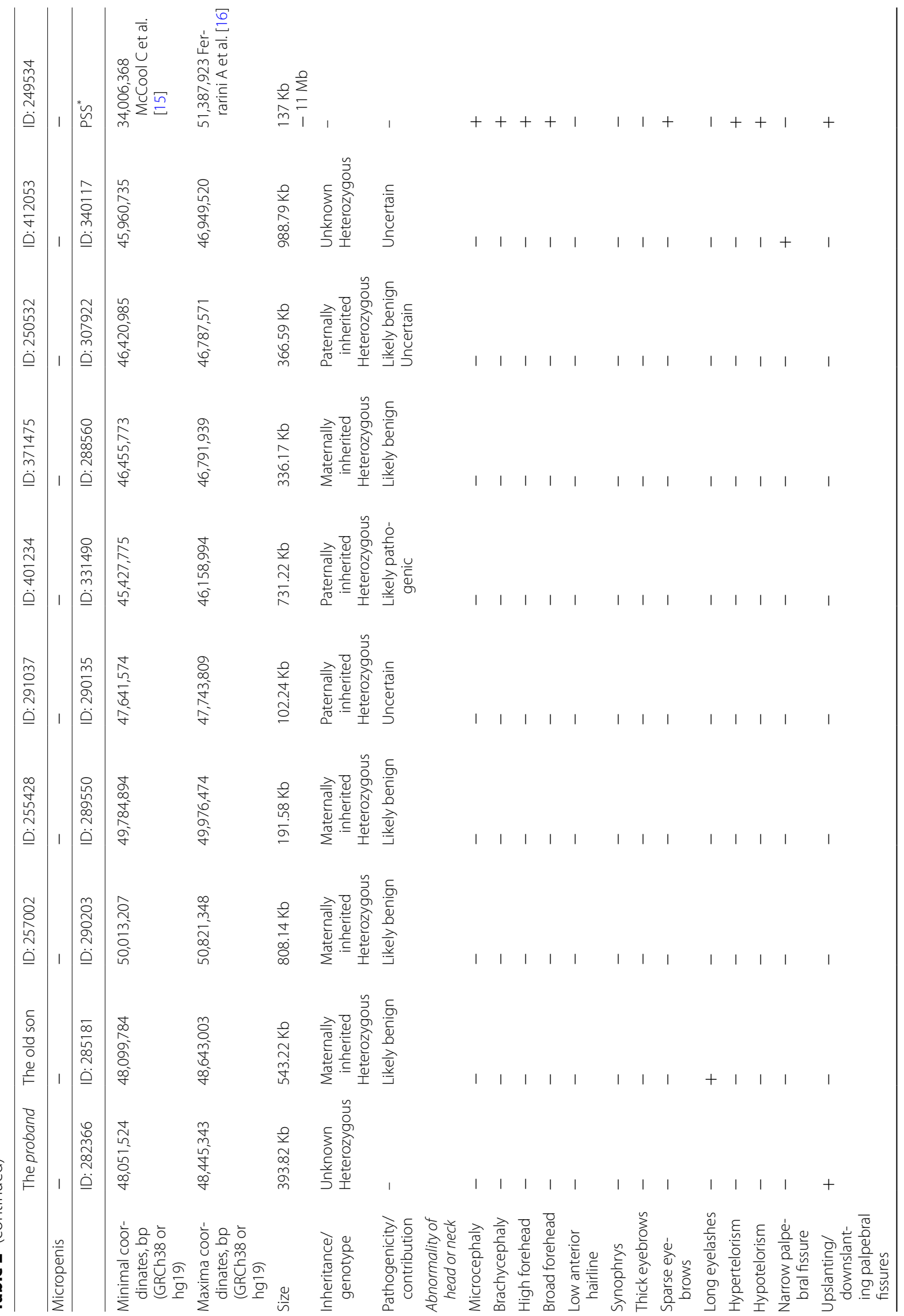




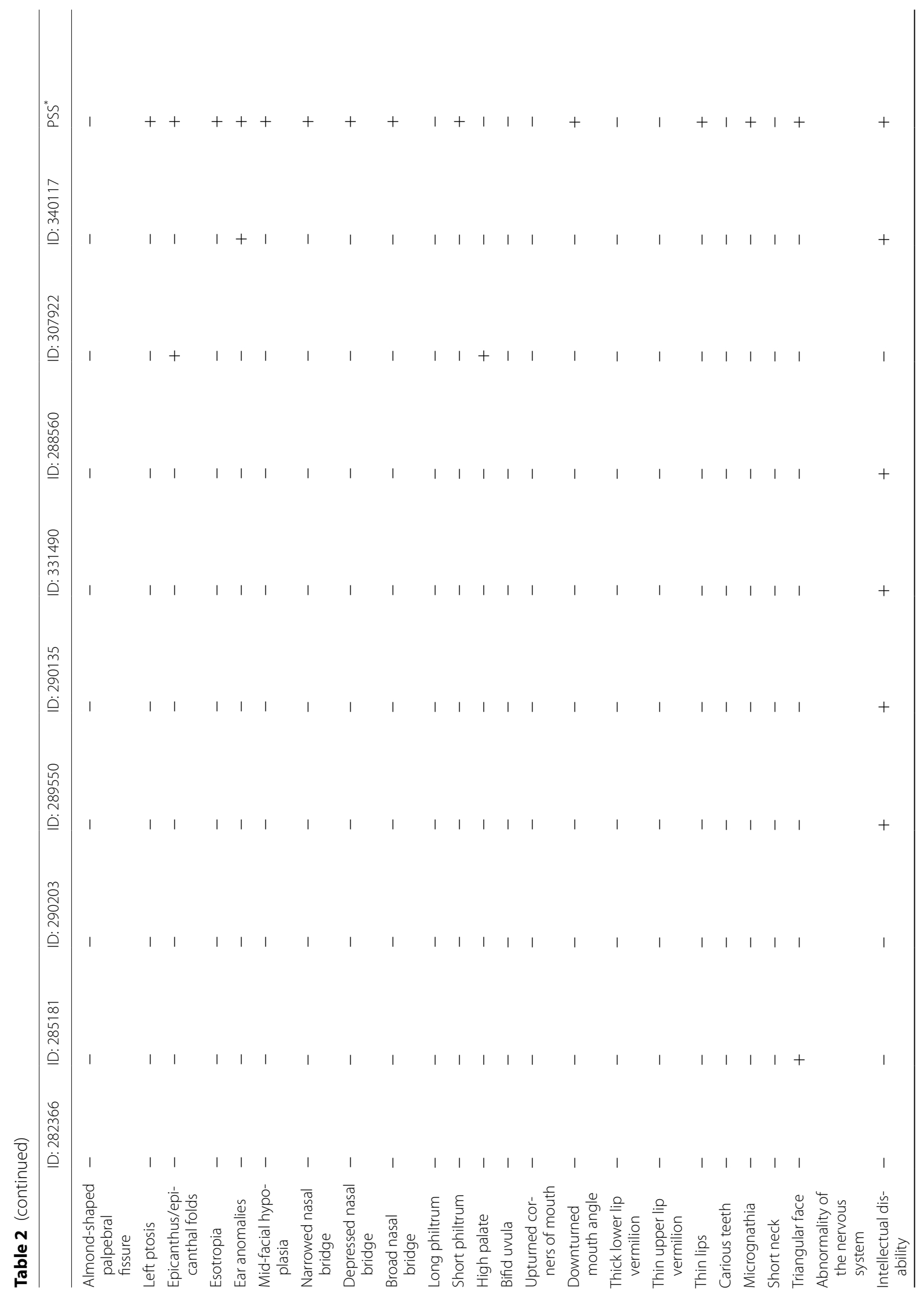




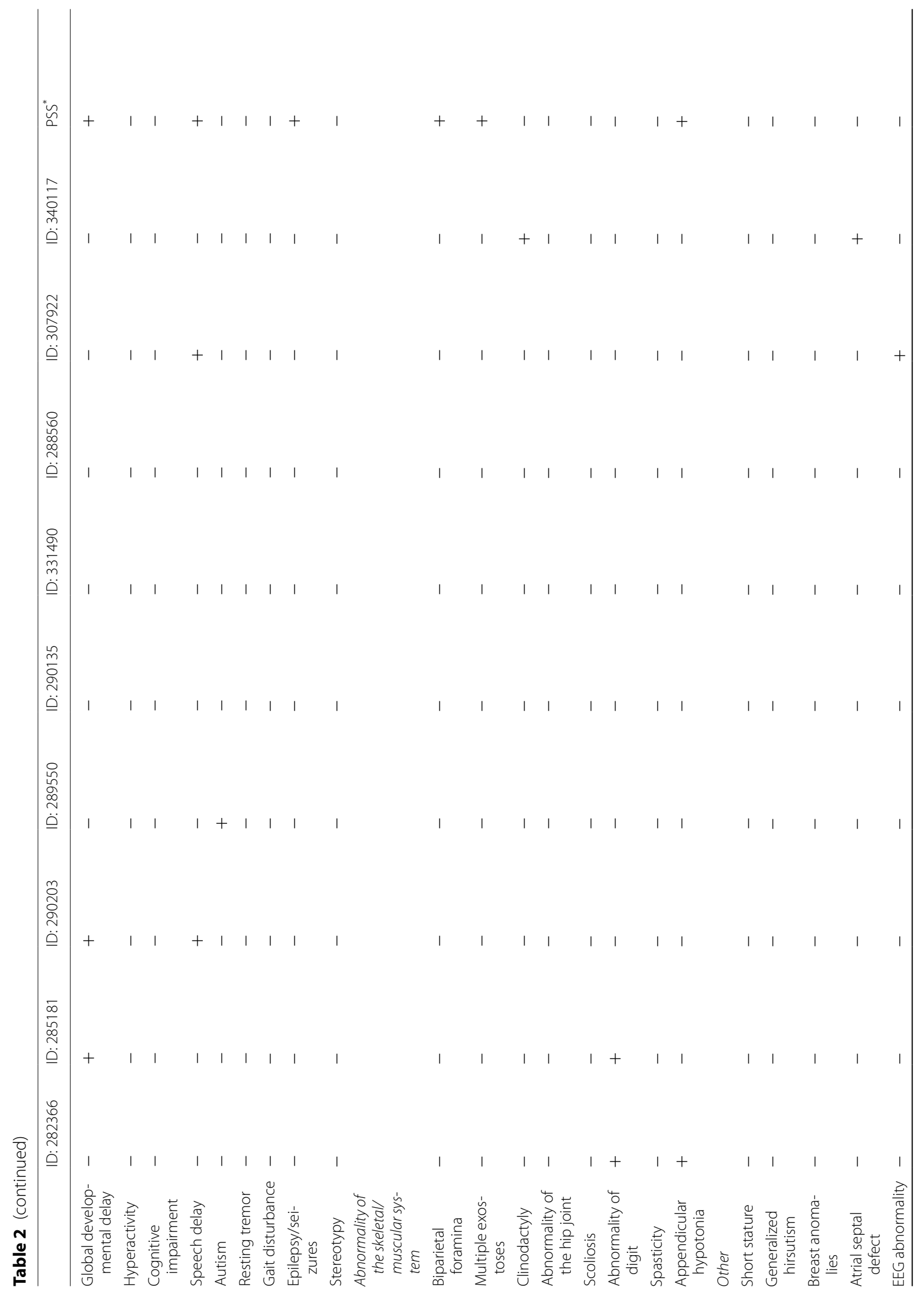




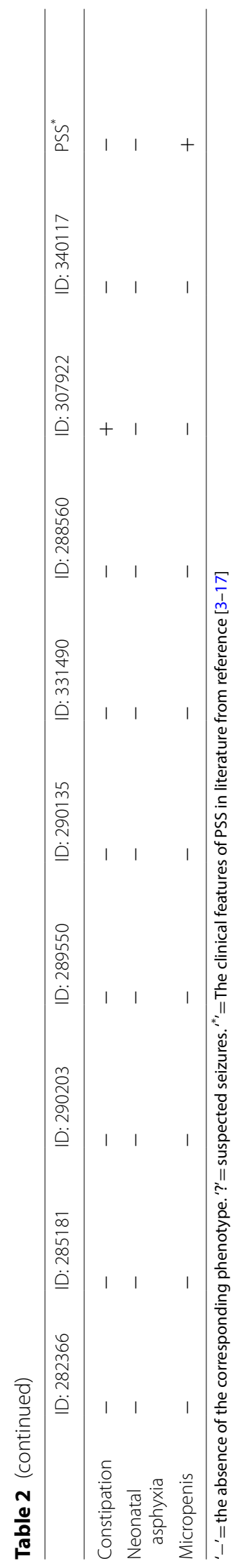




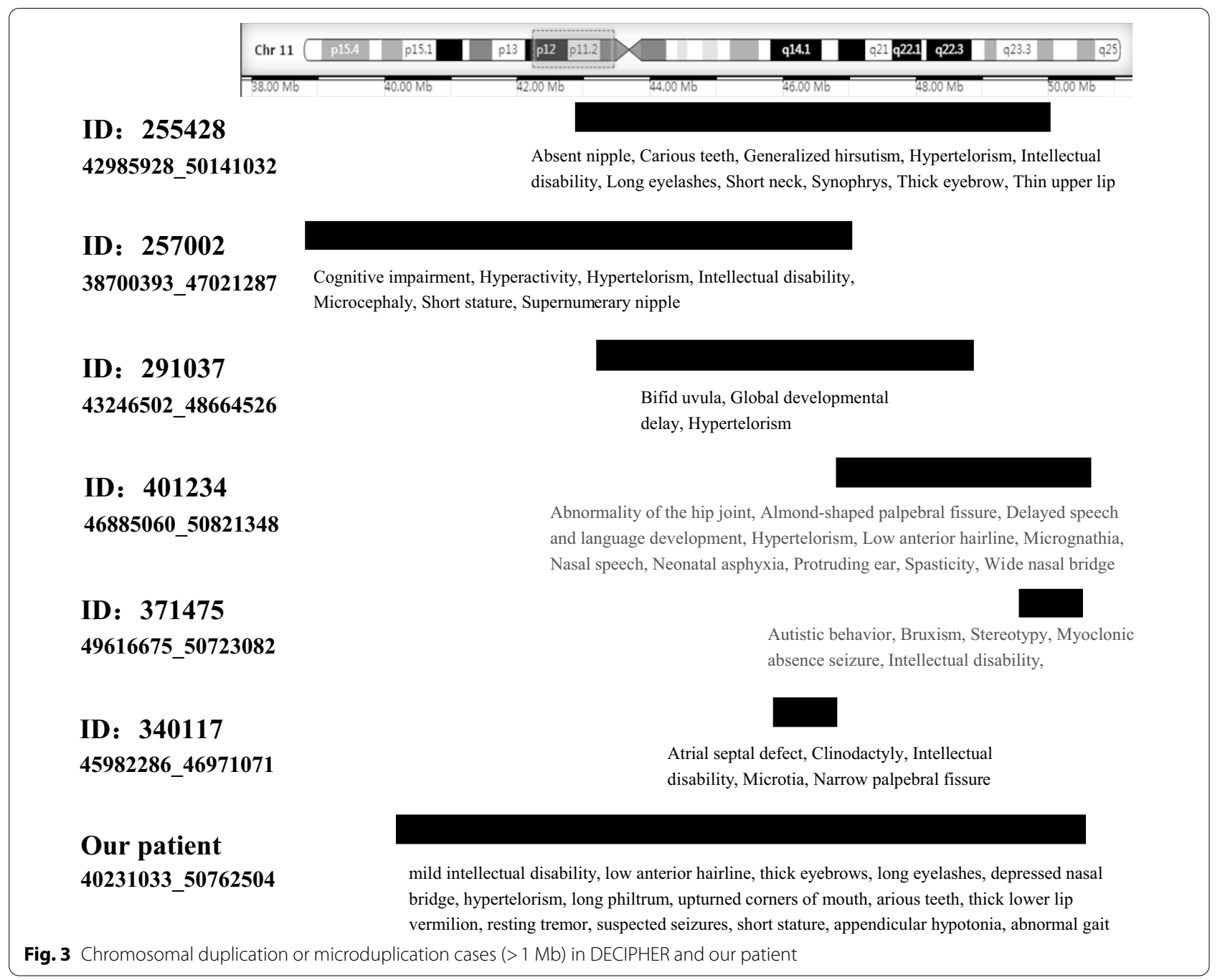

occurs in the brain [24]. PHF21A is the likely cause of ID and craniofacial abnormalities in Potocki-Shaffer Syndrome [25]. MAPK8IP1, also called Islet-brain-1 (IB1) or JNK-interacting protein-1 (JIP1), is abundantly expressed in the pancreas, brain, testis, and prostate and, to a lesser extent, in the heart, ovary, and small intestine [26]. HPA exhibits tissue-specific expression in the brain, with the strongest expression in the cerebral cortex. MAPK8IP1/JIP1 is a critical regulator of autophagosome transport in neurons, which ensures the fidelity of retrograde autophagosome transport in the axon and is highly sensitive to defects in autophagy [27]. JIP1 also plays an important role in neurons as a regulator of kinesin-1-dependent transport [28]. RAPSN, which is considered to be related to brain/cognition in DDG2P, shows low expression in HPA. Therefore, overexpression of this gene would not cause ID in this syndrome. $A L X 4$, SLC35C1, PHF21A, and MAPK8IP1 may be the genes most likely to have a gene-dosage effect in 11p11.12p12 duplication. Further work is required to fully elucidate the mechanisms leading to $11 \mathrm{p} 11.12 \mathrm{p} 12$ duplication. In ClinGen, there is no evidence for triplosensitive phenotypes for any of the above genes and only evidence for haploinsufficiency phenotypes. This study suggests that there are triplosensitive effect genes responsible for 11p11.12p12-related syndrome.

We present two patients in a family with de novo $11 \mathrm{p} 11.12 \mathrm{p} 12$ duplication and provide the results of five years of clinical follow-up. The duplication was identified and evaluated using a multidisciplinary protocol involving assessment by a geneticist, stomatologist, neurologist, psychiatrist, and paediatrician. The clinical features include mild ID, short stature, craniofacial anomalies, suspected seizures, resting tremor, appendicular hypotonia, and abnormal gait. The identification of clinical phenotypes will facilitate genetic counselling, especially prenatal genetic counselling. 


\section{Conclusions}

We report the first $11 \mathrm{p} 11.12 \mathrm{p} 12$ duplication in a family with ID and craniofacial abnormalities. The findings will facilitate the clinical diagnosis and genetic counselling of patients with 11p11.12p12 duplication in the future. Karyotype analysis and molecular cytogenetics were helpful to identify the genetic aetiology of the patients in the family and potential candidate genes with triplosensitive effects involved in $11 \mathrm{p} 11.12 \mathrm{p} 12$ duplication.

\begin{abstract}
Abbreviations
PSS: Potocki-Shaffer syndrome; EEG: Electroencephalogram; MRI: Magnetic resonance imaging; CMA: Chromosome microarray analysis; WES: Wholeexome sequencing; SNVs: Single nucleotide variations; ID: Intellectual disability; CFA: Craniofacial anomalies; ISCN: International System for Human Cytogenomic Nomenclature; PCR: Polymerase chain reaction; ChAS: Chromosome Analysis Suite; QC: Quality control; HGMD: Human Gene Mutation Database; OMIM: Online Mendelian Inheritance in Man; BWA: Burrows-Wheeler Aligner; DGV: Database of Genetic Variants; CT: Computed tomography; CNVs: Copy number variations; DECIPHER: Database of Chromosomal Imbalance and Phenotype in Humans using Ensembl Resources; FucT1: Fucose transmembrane transporter; BHC: BRAF35/histone deacetylase complex; RE1: Repressor element-1; NRS: Neural restrictive silencer; IB1: Islet-brain-1; JP1: JNK-interacting protein-1; SRA: Sequence Read Archive; GEO: Gene Expression Omnibus.
\end{abstract}

\section{Acknowledgements}

We are grateful to the patient and family for participating in this study. We also thank Guangzhou KingMed Diagnostics Group Co., Ltd. for technical assistances.

\section{Authors' contributions}

XJC, MZD, WWS, FW: designed and organized the study. XJC, HHX, WWS: drafted the manuscript. FW, BJC: acquired the clinical data. FFX, YZ, JG, XT, XJC, MZD, HHX, BJC: analysed and interpreted the experimental data. All authors read and approved the final manuscript.

\section{Funding}

This work was supported by a Grant from the Medical Science and Technology Project of Zhejiang Province (No. 2020KY349). The funding body played no role in the design of the study; the collection, analysis, and interpretation of data; or the writing of the manuscript.

\section{Availability of data and materials}

The datasets generated and/or analysed during the current study are available in the NCBI Sequence Read Archive (SRA) repository at https://www.ncbi.nlm. nih.gov/sra/ PRJNA713823, and the Gene Expression Omnibus (GEO) repository at https://www.ncbi.nlm.nih.gov/geo/query/acc.cgi?acc=GSE169469.

\section{Declarations}

\section{Ethics approval and consent to participate}

The present study was approved by the Ethics Committee of Taizhou Hospital of Zhejiang Province (Taizhou, China), and written informed consent was obtained from all patients prior to study commencement. Written informed consent was provided on behalf of minors by their parents.

\section{Consent for publication}

All patients signed written informed consent during the counselling sessions and agreed to share clinical details and clinical images in a reputable scientific journal. Written informed consent was also provided on behalf of the minors by their parents.

\section{Competing interests}

The authors declare that they have no competing interests.

\section{Author details}

${ }^{1}$ Medical Research Center, Taizhou Hospital of Zhejiang Province Affiliated to Wenzhou Medical University, Linhai, Zhejiang, China. ${ }^{2}$ Department of Neurology, Taizhou Hospital of Zhejiang Province Affiliated to Wenzhou Medical University, Linhai, Zhejiang, China. ${ }^{3}$ Department of Pharmacy, Taizhou Hospital of Zhejiang Province Affiliated to Wenzhou Medical University, Linhai, Zhejiang, China. ${ }^{4}$ Department of Public Research Platform, Taizhou Hospital of Zhejiang Province Affiliated to Wenzhou Medical University, Linhai, Zhejiang, China. ${ }^{5}$ Department of Mental Health, Taizhou Hospital of Zhejiang Province Affiliated to Wenzhou Medical University, Linhai, Zhejiang, China.

Received: 13 August 2020 Accepted: 24 March 2021

Published online: 09 April 2021

\section{References}

1. Bourke J, de Klerk N, Smith T, Leonard H. Population-based prevalence of intellectual disability and autism spectrum disorders in western Australia: a comparison with previous estimates. Medicine (Baltimore). 2016;95:e3737. https://doi.org/10.1097/MD.0000000000003737.

2. Erger F, Schaaf CP, Netzer C. Which genes to assess in the NGS diagnostics of intelectual disability? The case for a consensus database-driven and expert-curated approach. Mol Cell Probes. 2019;45:84-8. https://doi.org/ 10.1016/j.mcp.2019.03.006.

3. Shaffer LG, Hecht JT, Ledbetter DH, Greenberg F. Familial interstitial deletion 11 (p11.12p12) associated with parietal foramina, brachymicrocephaly, and mental retardation. Am J Med Genet. 1993;45:581-583. https:// doi.org/10.1002/ajmg.1320450512.

4. Potocki L, Shaffer LG. Interstitial deletion of 11(p11.2p12): a newly described contiguous gene deletion syndrome involving the gene for Hereditary Multiple Exostoses (EXT2). Am J Med Genet. 1996;62:319325.https://doi.org/10.1002/(SICI)1096-8628(19960329)62:3<319::AIDAJMG22>3.0.CO;2-M.

5. Chuang L, Wakui K, Sue WC, Su MH, Shaffer LG, Kuo PL. Interstitial deletion 11 (p11.12p11.2) and analphoid marker formation results in inherited Potocki-Shaffer syndrome. Am J Med Genet A. 2005;133A:180-183. https://doi.org/10.1002/ajmg.a.30362.

6. Swarr DT, Bloom D, Lewis RA, Elenberg E, Friedman EM, Glotzbach C, et al. Potocki-Shaffer syndrome: comprehensive clinical assessment, review of the literature, and proposals for medical management. Am J Med Genet A. 2010;152A:565-72. https://doi.org/10.1002/ajmg.a.33245.

7. Palka C, Alfonsi M, Mohn A, GuancialiFranchi P, Chiarelli F, Calabrese G. Delayed diagnosis of Potocki-Shaffer Syndrome in a woman with multiple exostoses and mental retardation. MolSyndromol. 2012;2:259-61. https://doi.org/10.1159/000337925.

8. Labonne JD, Vogt J, Reali L, Kong IK, Layman LC, Kim HG. A microdeletion encompassing PHF21A in an individual with global developmental delay and craniofaci ala- nomalies. Am J Med Genet A. 2015;167A:3011-3018. https://doi.org/10.1002/ajmg.a.37344.

9. Hamanaka K, Sugawara Y, Shimoji T, Nordtveit TI, Kato M, Nakashima M, et al. De novo truncating variants in PHF21A cause intellectual disability and craniofacial anomalies. Eur J Hum Genet. 2019;27:378-83. https://doi. org/10.1038/s41431-018-0289-X.

10. Kim HG, Kim HT, Leach NT, Lan F, Ullmann R, Silahtaroglu A, et al. Translocations disrupting PHF21A in the Potocki-Shaffer-syndrome region are associated with intellectual disability and craniofacial anomalies. Am J Hum Genet. 2012;91(1):56-72. https://doi.org/10.1016/j.ajhg.2012.05.005.

11. Cohen A. Intellectual disability and craniofacial anomalies explained: one more gene associated with Potocki-Shaffer syndrome. Clin Genet. 2012;82(6):522-3. https://doi.org/10.1111/cge.12002.

12. Sohn YB, Yim SY, Cho EH, Kim OH. The first Korean patient with PotockiShaffer syndrome: a rare cause of multiple exostoses. J Korean Med Sci. 2015;30(2):214-7. https://doi.org/10.3346/jkms.2015.30.2.214.

13. Kim HG, Rosenfeld JA, Scott DA, Bénédicte G, Labonne JD, Brown J, et al. Disruption of PHF21A causes syndromic intellectual disability with craniofacial anomalies, epilepsy, hypotonia, and neurobehavioral problems including autism. Mol Autism. 2019;10:35. https://doi.org/10.1186/ s13229-019-0286-0. 
14. Wissman SD, McCool C, Potocki L, Elenberg E. Hypertension in PotockiShaffer syndrome: a case report. Eur J Med Genet. 2020;63:103633. https://doi.org/10.1016/j.ejmg.2019.02.005.

15. McCool C, Spinks-Franklin A, Noroski LM, Potocki L. Potocki-Shaffer syndrome in a child without intellectual disability - the role of PHF21A in cognitive function. Am J Med Genet A. 2017;173:716-20. https://doi.org/ 10.1002/ajmg.a.37988

16. Ferrarini A, Gaillard M, Guerry F, Ramelli G, Heidi F, Keddache CV, et al. Potocki-Shaffer deletion encompassing ALX4 in a patient with frontonasal dysplasia phenotype. Am J Med Genet A. 2014;164A:346-52. https:// doi.org/10.1002/ajmg.a.36140.

17. Wakui K, Gregato G, Ballif BC, Glotzbach CD, Bailey KA, Kuo PL, et al. Construction of a natural panel of $11 \mathrm{p} 11.2$ deletions and further delineation of the critical region involved in Potocki-Shaffer syndrome. Eur J Hum Genet. 2005;13(5):528-540. https://doi.org/10.1038/sj.ejhg.5201366.

18. Joshi PA, Chang H, Hamel PA. Loss of Alx4, a stromally-restricted homeodomain protein, impairs mammary epithelial morphogenesis. Dev Biol. 2006;297:284-94. https://doi.org/10.1016/j.ydbio.2006.05.032.

19. Hussain S, Umm-E-Kalsoom, Ullah I, Liaqat K, Nawaz S, Ahmad W. A novel missense variant in the ALX4 gene underlies mild to severe frontonasal dysplasia in a consanguineous family. Genet Test Mol Biomark. 2020;24(4):217-223. https://doi.org/10.1089/gtmb.2019.0203.

20. Kayserili H, Uz E, Niessen C, Vargel I, Alanay Y, Tuncbilek G, et al. ALX4 dysfunction disrupts craniofacial and epidermal development. Hum Mol Genet. 2009;18:4357-66. https://doi.org/10.1093/hmg/ddp391.

21. Lühn K, Wild MK, Eckhardt M, Gerardy-Schahn R, Vestweber D. The gene defective in leukocyte adhesion deficiency II encodes a putative GDPfucose transporter. Nat Genet. 2001;28:69-72. https://doi.org/10.1038/ ng0501-69.

22. Deng M, Chen Z, Tan J, Liu H. Down-regulation of SLC35C1 induces colon cancer through over-activating Wnt pathway. J Cell Mol Med. 2020;24:3079-90. https://doi.org/10.1111/jcmm.14969.
23. Dauber A, Ercan A, Lee J, James P, Jacobs PP, Ashline DJ, et al. Congenital disorder of fucosylation type $2 \mathrm{c}$ (LADII) presenting with short stature and developmental delay with minimal adhesion defect. Hum Mol Genet. 2014;23:2880-7. https://doi.org/10.1093/hmg/ddu001.

24. Hakimi MA, Bochar DA, Chenoweth J, Lane WS, Mandel G, Shiekhattar R. A core-BRAF35 complex containing histone deacetylase mediates repression of neuronal-specific genes. Proc Natl Acad Sci USA. 2002;99:7420-5. https://doi.org/10.1073/pnas.112008599.

25. Porter RS, Murata-Nakamura Y, Nagasu H, Kim HG, Iwase S. Transcriptome analysis revealed impaired CAMP responsiveness in PHF21A-deficient human cells. Neuroscience. 2018;370:170-80. https://doi.org/10.1016/j. neuroscience.2017.05.031

26. Mooser V, Maillard A, Bonny C, Steinmann M, Shaw P, Yarnall DP, et al. Genomic organization, fine-mapping, and expression of the human isletbrain 1 (IB1)/C-Jun- amino-terminal kinase interacting protein-1 (JIP-1) gene. Genomics. 1999:55:202-8. https://doi.org/10.1006/geno.1998.5641.

27. Fu MM, Holzbaur EL. MAPK8IP1/JIP1 regulates the trafficking of autophagosomes in neurons. Autophagy. 2014;10:2079-81. https://doi. org/10.4161/auto.34451.

28. Nguyen TQ, Aumont-Nicaise M, Andreani J, Velours C, Chenon M, Vilela $F$, et al. Characterization of the binding mode of JNK-interacting protein 1 (JIP1) to kinesin- light chain 1 (KLC1). J BiolChem. 2018;293:13946-60. https://doi.org/10.1074/jbc.RA118.003916.

\section{Publisher's Note}

Springer Nature remains neutral with regard to jurisdictional claims in published maps and institutional affiliations.
Ready to submit your research? Choose BMC and benefit from:

- fast, convenient online submission

- thorough peer review by experienced researchers in your field

- rapid publication on acceptance

- support for research data, including large and complex data types

- gold Open Access which fosters wider collaboration and increased citations

- maximum visibility for your research: over $100 \mathrm{M}$ website views per year

At BMC, research is always in progress.

Learn more biomedcentral.com/submissions 